\title{
Covariance-Based Transmission Power Control for Estimation over Wireless Sensor Networks
}

\author{
Touraj Soleymani, Samuele Zoppi, Mikhail Vilgelm, \\ Sandra Hirche, Wolfgang Kellerer, and John S. Baras
}

\begin{abstract}
In this study, we would like to design a transmission power control mechanism for minimizing the transmit power of a wireless sensor node subject to a constraint on the quality of estimation at a remote estimator. In particular, we measure the estimation distortion by a mean square error function, and model the transmit power as a function of the packet success rate using the IEEE 802.15.4 standard which was formed for the specification of low-data-rate and low-power wireless communication. We formulate the problem with an infinite horizon discounted cost function. We show that there is a separation between the designs of the optimal estimator and optimal transmit power policy. Then, we use dynamic programming to characterize the optimal transmit power policy in terms of the estimation error covariance. Finally, we propose approximate value iteration as an approximation algorithm to calculate a near-optimal transmit power policy.
\end{abstract}

\section{INTRODUCTION}

Thanks to the recent advances in micro electro-mechanical systems (MEMS) and wireless communication technologies, the design and development of wireless sensor networks (WSNs) with small, low-cost, low-power, and multifunctional wireless sensor nodes have become feasible. In general, each node in a WSN consists of a sensor, a microprocessor, and a transceiver which communicates wirelessly in short distances. Applications of WSNs are many, in particular in healthcare, automation, and security (for more detail see [1] in which several WSN applications are described and the related challenges are explored).

A wireless sensor node can only be equipped with a limited power source due to severe hardware constraints. In addition, power source replacement is impossible for most applications, which makes lifetime of a node limited to lifetime of the battery. Among sensing, communication, and data processing, the power of a wireless sensor node is mainly consumed for communication. Hence, this motivates us to design WSN protocols and algorithms, apart from traditional performance metrics such as throughput and delay, based on energy efficiency. One effective technique to conserve energy

T. Soleymani and S. Hirche are with the chair of Information-Oriented Control, Technische Universität München, D-80333 Munich, Germany (email: $\{$ touraj, hirche\}@tum.de).

S. Zoppi, M. Vilgelm, and W. Kellerer are with the chair of Communication Networks, Technische Universität München, D-80333 Munich, Germany (email: \{samuele.zoppi, mikhail.vilgelm, wolfgang.kellerer\}etum. de).

J. S. Baras is with the Institute for Systems Research, University of Maryland College Park, MD 20742, USA (email: baras @umd.edu).

T. Soleymani, S. Hirche, and J. S. Baras are also with the Institute for Advanced Study, Technische Universität München, D-85748 Garching, Germany. in a wireless sensor node is transmission power control (TPC) [2].

In this study, we would like to design a TPC mechanism for minimizing the transmit power of a wireless sensor node subject to a constraint on the quality of estimation at a remote estimator. In fact, the transmit power influences the signalto-noise ratio and subsequently the packet dropout rate. It is shown in [3] that packet dropouts have a negative impact on the performance of the remote estimation. Therefore, we need an effective TPC mechanism that considers the task performance as well as the realistic hardware features and constraints of the wireless sensor node. Intuitively speaking, this TPC mechanism should adaptively adjust the transmit power such that a high level of transmit power is used only for measurements with important information.

In [2], the problem of power management in WSNs is discussed in detail and various TPC mechanisms are studied. It is highlighted there that a TPC mechanism employed at the medium access control (MAC) layer significantly impacts the power consumption of the wireless sensor nodes. Recently, different TPC mechanisms have been proposed in the literature for the estimation problem over wireless channels. A covariance-based TPC mechanism for the estimation outage minimization problem over a fading channel is investigated in [4]. A measurement-based TPC mechanism for estimation over a fading channel when the state estimate is transmitted is proposed in [5]. A study on the joint design of an estimator and a TPC mechanism for estimation (without considering the measurement noise) over a fading channel with an infinite horizon average cost function is carried out in [6]. In addition, the results are extended in [7] and [8] by taking into account the power constraints imposed by the energy harvesting.

In this paper, we develop a framework for achieving the minimum expected transmit power that satisfies a specific level of distortion in the estimation problem of a linear dynamical system over a wireless communication channel. We take a practical approach by selecting a realistic channel model and a specific physical layer modulation compliant with the state-of-the-art WSN hardware platforms. In particular, we measure the estimation distortion by a mean square error function, and model the transmit power as a function of the packet success rate using the IEEE 802.15.4 standard which was formed for the specification of low-datarate and low-power wireless communication. We formulate the problem with an infinite horizon discounted cost function. We show that there is a separation between the designs of 
the optimal estimator and optimal transmit power policy. Then, we use dynamic programming to characterize the optimal transmit power policy in terms of the estimation error covariance. Finally, we propose approximate value iteration as an approximation algorithm to calculate a near-optimal transmit power policy.

The outline of this paper is as follows. Problem formulation is presented in Section II. In Section III, we derive the optimal estimator and optimal transmit power policy, and show a separation between their designs. In Section IV, we propose an approximate algorithm for calculating a nearoptimal transmit power policy. We illustrate numerical and simulation results in Section V. Finally, concluding remarks are made in Section VI.

\section{A. Notations}

In this paper, we represent an $n$ dimensional vector with $x=\left[x_{1}, \ldots, x_{n}\right]^{T}$ where $x_{i}$ is its $i$ th component. We write $x^{T}$ to denote the transpose of the vector $x$. The normal distribution with mean $\mu$ and covariance $\Sigma$ is denoted by $N(\mu, \Sigma)$. The expected value and covariance of the random variable $x$ are denoted by $\mathrm{E}[x]$ and $\operatorname{cov}[x]$ respectively. For matrices $A$ and $B$, we write $A \succ 0$ and $B \succeq 0$ to mean that $A$ and $B$ are positive definite and positive semi-definite respectively.

\section{PRoblem Formulation}

Consider a discrete-time dynamical system generated by the following linear state equation:

$$
x_{k+1}=F x_{k}+w_{k}
$$

for time $k \in \mathbb{N}_{+}$and with initial condition $x_{0}$ where $x_{k} \in \mathbb{R}^{n}$ is the state of the system, $F$ is the state matrix, $w_{k} \in \mathbb{R}^{n}$ is a Gaussian white noise with zero mean and covariance $R_{1}$ where $R_{1} \succ 0$. At each time step, the output of the system is measured by a wireless sensor node. The measurement of the sensor is given by

$$
y_{k}=H x_{k}+v_{k}
$$

where $y_{k} \in \mathbb{R}^{p}$ is the output of the system, $H$ is the output matrix, and $v_{k} \in \mathbb{R}^{p}$ is a Gaussian white noise with zero mean and covariance $R_{2}$ where $R_{2} \succ 0$. It is assumed that the initial state $x_{0}$ is a Gaussian vector with mean $m$ and covariance $R_{0}$, and that $x_{0}, w_{k}$, and $v_{k}$ are mutually independent. In addition, it is assumed that $(F, H)$ is observable.

The wireless sensor node is connected to a remote estimator via a wireless communication channel which is unreliable and modeled by a one-step-delay packet-erasure channel with acknowledgement. The packet loss in the channel is modeled according to a Bernoulli arrival process $\gamma_{k}$ with mean equal to the packet success rate $\mathrm{PSR}_{k}$ such that

$$
\gamma_{k}= \begin{cases}1, & \text { if packet is received successfully } \\ 0, & \text { otherwise }\end{cases}
$$

Assuming all possible errors in a packet can be detected, the packet success rate for transmission of a packet with $\ell$ bits is given by

$$
\mathrm{PSR}_{k}=\left(1-\mathrm{BER}_{k}\right)^{\ell},
$$

where $\mathrm{BER}_{k}$ is the bit error rate. Following the offset quadrature phase-shift keying (O-QPSK) modulation [9] used in IEEE 802.15.4 we have

$$
\mathrm{BER}_{k}=Q\left(\sqrt{\frac{2 B_{N}}{R_{c}} \overline{\mathrm{SNR}}_{k}}\right)
$$

where $Q($.$) denotes the Q-function, B_{N}$ is the bandwidth of the noise, $R_{c}$ is the data rate of the communication, and $\overline{\mathrm{SNR}}_{k}$ is the average signal-to-noise ratio. Inserting (5) in (4), we obtain

$$
\mathrm{PSR}_{k}=\left(1-Q\left(\sqrt{\frac{2 B_{N}}{R_{c}} \overline{\mathrm{SNR}}_{k}}\right)\right)^{\ell}
$$

Hence, the required average signal-to-noise ratio for a given packet success rate is obtained by

$$
\overline{\mathrm{SNR}}_{k}=\frac{R_{c}}{2 B_{N}}\left(Q^{-1}\left(1-\mathrm{PSR}_{k}^{\frac{1}{\ell}}\right)\right)^{2} .
$$

In addition, every transmission experiences a channel realization specified by considering path loss and shadowing effects. The received power for a transmission is obtained by

$$
p_{k}^{R X}=p_{k}^{T X} p_{L}^{-1} p_{S}^{-1}
$$

where $p_{k}^{R X}$ is the received power, $p_{k}^{T X}$ is the transmit power, and $p_{L}$ and $p_{S}$ capture the amount of path loss and shadowing of a particular environment respectively given by

$$
\begin{aligned}
& p_{L}=\left(\frac{4 \pi f d_{0}}{c}\right)^{2}\left(\frac{d}{d_{0}}\right)^{\eta}, \\
& \ln p_{S} \sim N\left(0, \sigma_{S}^{2}\right),
\end{aligned}
$$

where $d_{0}$ is the reference distance, $d$ is the receiver distance, $f$ is the carrier frequency, $c$ is the speed of light, $\eta$ is the path loss exponent, and $\sigma_{S}^{2}$ is the shadowing variance. Notice that the shadowing is modeled with a log-normal distribution which is suitable for short-range indoor propagation. Then, for each transmission the resulting signal-to-noise ratio is obtained by

$$
\mathrm{SNR}_{k}=\frac{p_{k}^{R X}}{p_{N}}=\frac{p_{k}^{T X}}{p_{N} p_{L} p_{S}}
$$

where $p_{N}$ is the noise power floor. Taking the expectation, we can obtain the transmit power as a function of the average signal-to-noise ratio:

$$
p_{k}^{T X}=p_{N}\left(\frac{4 \pi f d_{0}}{c}\right)^{2}\left(\frac{d}{d_{0}}\right)^{\eta} e^{\frac{\sigma_{S}^{2}}{2}} \overline{\mathrm{SNR}}_{k}
$$

Using (7) and (12), the required transmit power for a given packet success rate is obtained as

$$
p_{k}^{T X}= \begin{cases}c_{0}\left(Q^{-1}\left(1-\mathrm{PSR}_{k}^{\frac{1}{\ell}}\right)\right)^{2}, & \mathrm{PSR}_{k} \in[a, b], \\ 0, & \mathrm{PSR}_{k}=0 .\end{cases}
$$




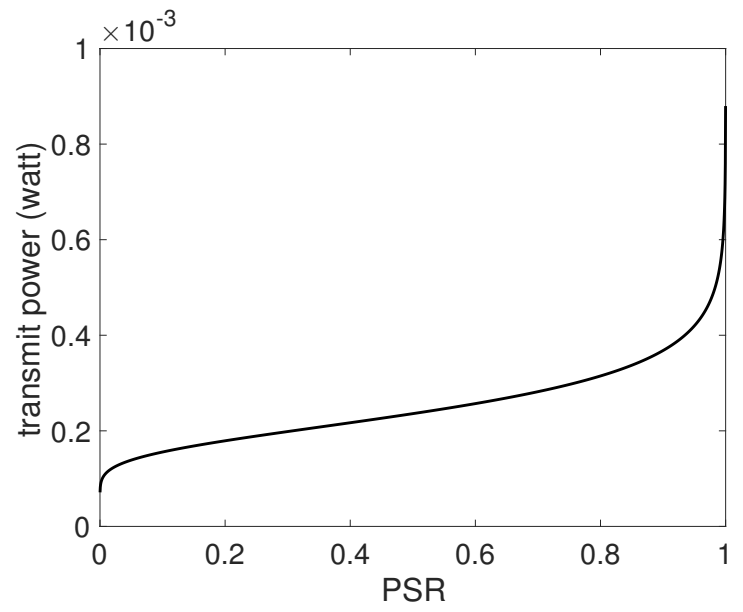

Fig. 1. The required transmit power as a function of the packet success rate for the specific wireless channel described in Section V.

where $[a, b]$ represents the operational range of $\mathrm{PSR}_{k}$ with specific $a$ and $b$, and

$$
c_{0}=\frac{R_{c}}{2 B_{N}} p_{N}\left(\frac{4 \pi f d_{0}}{c}\right)^{2}\left(\frac{d}{d_{0}}\right)^{\eta} e^{\frac{\sigma_{S}^{2}}{2}} .
$$

With abuse of notation we consider $\mathrm{PSR}_{k}=0$ for the case in which the transmitter is in sleep mode. Therefore, if $\mathrm{PSR}_{k}=$ 0 then nothing is transmitted at time $k$. In the sequel, we denote the function in (13) by $p_{k}^{T X}=\psi\left(\mathrm{PSR}_{k}\right)$ defined in the domain $\Gamma=0 \cup[a, b]$.

The required transmit power as a function of the packet success rate is depicted in Figure 1 for the specific wireless channel described in Section V.

Let the available information at the remote estimator at time $k$ be specified by:

$$
\mathcal{I}_{k}=\left\{\gamma_{0: k-1}, y_{0: k-1}\right\}
$$

We measure the estimation distortion by a mean square error function:

$$
\Phi=\mathrm{E}\left[\sum_{k=0}^{N}\left\|x_{k}-\hat{x}_{k}\right\|_{\Theta_{k}}^{2}\right]
$$

where $\Theta_{k} \succ 0$ is a weighting matrix and $\hat{x}_{k}$ is the best state estimate given the information set $\mathcal{I}_{k}$.

Let $\mu$ be a transmit power policy. We would like to achieve the minimum expected transmit power that satisfies a specific level of distortion $\beta$, i.e.,

$$
\Psi=\inf _{\mu: \Phi \leq \beta} \mathrm{E}\left[\sum_{k=0}^{N} p_{k}^{T X}\right]
$$

which can equivalently be written as

$$
\Psi=\inf _{\mu} \mathrm{E}\left[\sum_{k=0}^{N}\left(p_{k}^{T X}+\lambda\left\|x_{k}-\hat{x}_{k}\right\|_{\Theta_{k}}^{2}\right)\right],
$$

for a $\lambda>0$. In this paper, we study this problem over an infinite horizon with a discounted cost function expressed by

$$
\Psi_{\infty}=\inf _{\mu} \lim _{N \rightarrow \infty} \mathrm{E}\left[\sum_{k=0}^{N} \alpha^{k}\left(p_{k}^{T X}+\lambda\left\|x_{k}-\hat{x}_{k}\right\|_{\Theta_{k}}^{2}\right)\right],
$$

where $\alpha \in(0,1)$ is a discount factor which weighs the relative contribution of the costs in the short-term and longterm future.

\section{Optimal Transmit Power Policy}

The estimation is performed at the remote estimator based on the information set $\mathcal{I}_{k}$. The following lemma provides the best state estimate at the remote estimator.

Lemma 1: The conditional expected value of the state with the following dynamics is the minimizer of the distortion function in (16) for the system in (1) and (2) over the one-step-delay packet-erasure channel with the arrival process in (3):

$$
\begin{aligned}
& \hat{x}_{k+1}=F \hat{x}_{k}+\gamma_{k} K_{k}\left(y_{k}-H \hat{x}_{k}\right), \\
& P_{k+1}=F P_{k} F^{T}+R_{1}-\gamma_{k} K_{k} H P_{k} F^{T},
\end{aligned}
$$

where $\hat{x}_{k}=\mathrm{E}\left[x_{k} \mid \mathcal{I}_{k}\right], P_{k}=\operatorname{cov}\left[x_{k} \mid \mathcal{I}_{k}\right]$, and

$$
K_{k}=F P_{k} H^{T}\left(H P_{k} H^{T}+R_{2}\right)^{-1},
$$

with initial conditions $\hat{x}_{0}=m_{0}$ and $P_{0}=R_{0}$.

Proof: Since the conditional distribution of $x_{k}$ given $\mathcal{I}_{k}$ is Gaussian and the distortion is measured by a mean square error function, the conditional expectation $\mathrm{E}\left[x_{k} \mid \mathcal{I}_{k}\right]$ is the best state estimate. Following the Kolmogorov forward equation [10], the estimate and its covariance are propagated as

$$
\begin{aligned}
& \hat{x}_{k+1}=F \hat{x}_{k^{+}}, \\
& P_{k+1}=F P_{k^{+}} F^{T}+R_{1},
\end{aligned}
$$

where $k^{+}$denotes time $k$ just after the update of the estimate and its covariance. From Bayes' rule [10], the estimate and its covariance are updated as

$$
\begin{aligned}
& \hat{x}_{k^{+}}=\hat{x}_{k}+\gamma_{k} P_{k} H^{T}\left(H P_{k} H^{T}+R_{2}\right)^{-1}\left(y_{k}-H \hat{x}_{k}\right), \\
& P_{k^{+}}=\left(I_{n}-\gamma_{k} P_{k} H^{T}\left(H P_{k} H^{T}+R_{2}\right)^{-1} H\right) P_{k} .
\end{aligned}
$$

We obtain the result by substituting (22) and (23) in (20) and (21) respectively.

Let us use the identity $\mathrm{E}\left[\left\|x_{k}-\hat{x}_{k}\right\|_{\Theta_{k}}^{2}\right]=\mathrm{E}\left[\operatorname{tr}\left(\Theta_{k} P_{k}\right)\right]$. Then, the optimal transmit power policy is obtained by solving the following optimization problem:

$$
\begin{array}{ll}
\min . & \lim _{N \rightarrow \infty} \mathrm{E}\left[\sum_{k=0}^{N} \alpha^{k} p_{k}^{T X}+\alpha^{k} \lambda \operatorname{tr}\left(\Theta_{k} P_{k}\right)\right], \\
\text { s.t. } & P_{k+1}=F P_{k} F^{T}+R_{1}-\gamma_{k} K_{k} H P_{k} F^{T}, \\
& p_{k}^{T X}=\psi\left(\mathrm{PSR}_{k}\right),
\end{array}
$$

with initial condition $P_{0}=R_{0}$.

Theorem 1: There is a separation between the designs of the optimal estimator and optimal transmit power policy. 
Proof: The proof follows from the above derivations. The best state estimate is obtained by the recursive filter given in (19) and the optimal transmit power policy is obtained by solving the optimization problem in (24).

Next, we shall use dynamic programming in order to characterize the solution of the optimization in (24). We can eliminate the transmit power equality, and obtain the following equivalent optimization problem:

$$
\begin{aligned}
& \min . \lim _{N \rightarrow \infty} \mathrm{E}\left[\sum_{k=0}^{N} \alpha^{k} \psi\left(\mathrm{PSR}_{k}\right)+\alpha^{k} \lambda \operatorname{tr}\left(\Theta_{k} P_{k}\right)\right], \\
& \text { s.t. } P_{k+1}=F P_{k} F^{T}+R_{1}-\gamma_{k} K_{k} H P_{k} F^{T} .
\end{aligned}
$$

We denote the optimal solution of the above optimization problem by $J^{*}\left(P_{0}\right)$. Let $\pi$ be a packet success rate policy. We would like to find the optimal stationary packet success rate policy $\pi^{*}$. Then, the optimal transmit power policy can be obtained by

$$
\mu^{*}=\psi\left(\pi^{*}\right)
$$

Let us define

$$
\rho\left(P_{k}, \mathrm{PSR}_{k}\right)=\psi\left(\mathrm{PSR}_{k}\right)+\lambda \operatorname{tr}\left(\Theta_{k} P_{k}\right)
$$

and

$$
\phi\left(P_{k}, \mathrm{PSR}_{k}\right)=F P_{k} F^{T}+R_{1}-\gamma_{k} K_{k} H P_{k} F^{T} .
$$

The optimization problem in (25) can concisely be rewritten as

$$
\begin{array}{ll}
\min . & \lim _{N \rightarrow \infty} \mathrm{E}\left[\sum_{k=0}^{N} \alpha^{k} \rho\left(P_{k}, \mathrm{PSR}_{k}\right)\right] \\
\text { s.t. } & P_{k+1}=\phi\left(P_{k}, \mathrm{PSR}_{k}\right),
\end{array}
$$

with initial condition $P_{0}=R_{0}$. The next theorem shows that the optimal cost function satisfies the Bellman's equation.

Theorem 2: The optimal cost function $J^{*}(P)$ satisfies

$$
J^{*}(P)=T J^{*}(P),
$$

where $T$ is the dynamic programming operator defined as

$$
T J(P)=\min _{\mathrm{PSR} \in \Gamma} \mathrm{E}[\rho(P, \mathrm{PSR})+\alpha J(\phi(P, \mathrm{PSR}))] .
$$

Proof: Notice that $\rho(P, \mathrm{PSR}) \geq 0$. Then, the proof follows from the dynamic programming principle for infinite horizon discounted cost functions with positive stage costs. Refer to [11].

The following proposition shows the convergence of the dynamic programming recursive equation.

Proposition 1: For any function $J(P)$, the operator $T^{k} J(P)$ is convergent, i.e.,

$$
J_{\infty}(P)=J^{*}(P),
$$

where $J_{\infty}(P)=\lim _{k \rightarrow \infty} T^{k} J(P)$.

Proof: Please refer to [11].

The next proposition provides the sufficient and necessary condition for the optimality of a stationary packet success rate policy.
Proposition 2: The stationary packet success rate policy $\pi$ is optimal if and only if

$$
T_{\pi} J^{*}(P)=T J^{*}(P),
$$

where the operator $T_{\pi}$ is defined as

$$
T_{\pi} J(P)=\mathrm{E}[\rho(P, \pi)+\alpha J(\phi(P, \pi))] .
$$

Proof: Please refer to [11].

Finally, the next proposition gives the optimal stationary packet success rate policy based on the value function.

Proposition 3: The optimal stationary packet success rate policy is given by

$$
\begin{array}{r}
\mathrm{PSR}^{*}=\underset{\mathrm{PSR} \in \Gamma}{\operatorname{argmin}}\left\{\rho(P, \mathrm{PSR})+\alpha(1-\mathrm{PSR}) J^{*}\left(F P F^{T}+R_{1}\right)\right. \\
\left.+\alpha \mathrm{PSR}^{*}\left(F P F^{T}+R_{1}-K_{k} H P F^{T}\right)\right\},
\end{array}
$$

where $J^{*}(P)$ is the value function and satisfies (30).

Proof: Since the stage cost $\rho(P, \mathrm{PSR})$ for each $P$ is deterministic, from Theorem 2 we have

$$
T J^{*}(P)=\min _{\mathrm{PSR} \in \Gamma}\left\{\rho(P, \mathrm{PSR})+\alpha \mathrm{E}\left[J^{*}(\phi(P, \mathrm{PSR}))\right]\right\} .
$$

Moreover, the transition function $\phi(P, \mathrm{PSR})$ for each $P$ and any PSR can only take two different values:

$$
\begin{aligned}
& P_{+}^{0}=F P_{k} F^{T}+R_{1}, \\
& P_{+}^{1}=F P_{k} F^{T}+R_{1}-K_{k} H P_{k} F^{T} .
\end{aligned}
$$

The probability of $P_{k+1}=P_{+}^{1}$ is PSR and the probability of $P_{k+1}=P_{+}^{0}$ is $1-\mathrm{PSR}$. Therefore, we can calculate the expected value of the value function as

$$
\mathrm{E}\left[J^{*}(\phi(P, \mathrm{PSR}))\right]=(1-\mathrm{PSR}) J^{*}\left(P_{+}^{0}\right)+\operatorname{PSR} J^{*}\left(P_{+}^{1}\right) .
$$

Using Proposition 2, we have

$$
\begin{gathered}
T_{\pi} J^{*}(P)=\min _{\mathrm{PSR} \in \Gamma}\left\{\rho(P, \mathrm{PSR})+\alpha(1-\mathrm{PSR}) J^{*}\left(P_{+}^{0}\right)\right. \\
\left.+\alpha \mathrm{PSR} J^{*}\left(P_{+}^{1}\right)\right\} .
\end{gathered}
$$

The optimal PSR is then given by the argument of this minimization.

\section{Approximate Algorithm}

The spaces of the covariance and packet success rate are infinite. In order to use a computational method to solve the dynamic programming recursive equation given by (30), we approximate the problem via discretization. We discretize the packet success rate space by the following finite grid:

$$
S_{1}=\left\{\mathrm{PSR}^{1}, \mathrm{PSR}^{2}, \ldots, \mathrm{PSR}^{M_{1}}\right\}
$$

where $M_{1}$ denotes the cardinality of $S_{1}, \mathrm{PSR}^{1}=0$, and $a \leq \mathrm{PSR}^{i} \leq b$ for $i \geq 2$. To discretize the covariance space, we use the following finite grid:

$$
S_{2}=\left\{P^{1}, P^{2}, \ldots, P^{M_{2}}\right\},
$$


where $M_{2}$ denotes the cardinality of $S_{2}$ and $0 \preceq P^{i} \prec \infty$. We express the non-grid covariances by a linear interpolation of grid covariances, i.e.,

$$
P=\sum_{i=1}^{M_{2}} w_{i}(P) P^{i}
$$

where weights $w_{i}$ are nonnegative. Given that $J\left(P^{i}\right)$ is the value function of a grid covariance $P^{i}$, the value function of a non-grid covariance $P$ is approximated by

$$
\tilde{J}(P)=\sum_{i=1}^{M_{2}} w_{i}(P) J\left(P^{i}\right) .
$$

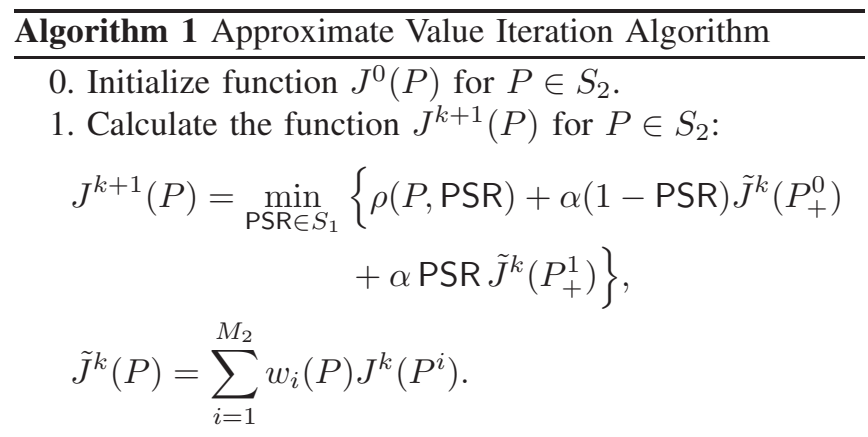

2. Stop if $\max _{P \in S_{2}}\left|J^{k+1}(P)-J^{k}(P)\right|<\epsilon_{0}$; otherwise assign $k:=k+1$ and go to Step 1 .

We use approximate value iteration, described in Algorithm 1 , to compute a near-optimal stationary packet success rate policy. In approximate value iteration, we generate a sequence of functions $J^{k}(P)$ which are approximations of the functions $T^{k} J(P)$. In each iteration, the improved function $J^{k+1}(P)$ is obtained based on the previous function $J^{k}(P)$. The algorithm stops when the maximum difference between two successive functions is less than a specific tolerance $\epsilon_{0}$. Let $k_{s}$ denote the step Algorithm 1 stops. We obtain the near-optimal stationary packet success rate policy as

$$
\begin{aligned}
\operatorname{PSR}^{*}(P)=\underset{\mathrm{PSR} \in S_{1}}{\operatorname{argmin}}\{ & \rho(P, \mathrm{PSR})+\alpha(1-\mathrm{PSR}) \tilde{J}^{k_{s}}\left(P_{+}^{0}\right) \\
& \left.+\alpha \mathrm{PSR} \tilde{J}^{k_{s}}\left(P_{+}^{1}\right)\right\}
\end{aligned}
$$

where $P_{+}^{0}$ and $P_{+}^{1}$ are defined in (36) and (37).

The following proposition gives the optimality bound of the approximate value iteration algorithm.

Proposition 4: Assume that

$$
\max _{P \in S_{2}}\left|J^{k+1}(P)-T J^{k}(P)\right| \leq \epsilon, \quad k \in \mathbb{N}_{+} .
$$

Then,

$$
\max _{P \in S_{2}}\left|J_{\infty}(P)-J^{*}(P)\right| \leq \frac{\epsilon}{1-\alpha} .
$$

Proof: Please refer to [11].

\section{ILLUSTRATIVE EXAMPLE}

In this section, we provide a simple example to show how our framework can be used to obtain the optimal stationary transmit power policy. Consider the following unstable system observed by a wireless sensor node:

$$
\begin{aligned}
x_{k+1} & =1.01 x_{k}+w_{k}, \\
y_{k} & =0.3 x_{k}+v_{k},
\end{aligned}
$$

where the covariances are $R_{1}=0.1$ and $R_{2}=0.5$. The state of the system is to be estimated by a remote estimator which receives the measurements from the wireless sensor node through a one-step-delay packet-erasure channel with acknowledgement. The communication channel is characterized by the following parameters: the bandwidth of the noise $B_{N}=2 \mathrm{MHz}$, the data rate of the communication $R_{c}=250 \mathrm{Kbps}$, the carrier frequency $f=2.4 \mathrm{GHz}$, the reference distance $d_{0}=1 \mathrm{~m}$, the receiver distance $d=10 \mathrm{~m}$, the path loss exponent $\eta=3.6$, the shadowing variance $\sigma_{S}^{2}=8$, the noise power floor $p_{N}=1.2 \times 10^{-11}$ watt, and the packet length $\ell=100$ bits. This yields $c_{0}=3.8929 \times 10^{-5}$. We choose the weighting matrix $\Theta=1$, the discount factor $\alpha=0.95$, and the weighting coefficient $\lambda=0.0007$.

The space of the packet success rate is discretized by a grid with cardinality $M_{1}=2500$ with $a=0.0001$ and $b=0.9999$, and the space of the covariance is discretized by a grid with cardinality $M_{2}=2500$ over the interval $[0,10]$. We consider $\mathrm{PSR}^{1}=0$ for the case in which the transmitter is in sleep mode. We used the approximate value iteration algorithm given in Algorithm 1 with $\epsilon_{0}=10^{-5}$ to approximate the optimal cost function $J^{*}(P)$. Then, we calculated the near-optimal stationary transmit power policy.

Figure 2 illustrates the near-optimal stationary transmit power policy as a function of the covariance. As it is seen, the transmitter is in sleep mode for small values of the covariance. This result can justify usage of a simple threshold event-triggering mechanism for transmission power control (see e.g., [12]). We assume that the initial conditions of the system are $x_{0}=1, \hat{x}_{0}=0$, and $P_{0}=2$. We simulated the system over the horizon $N=200$. Figure 3 and Figure 4 depict the trajectories of the estimation error and covariance respectively, and Figure 5 illustrates the trajectory of the transmit power. It can be observed that the transmit power is adaptive and at many time steps the transmitter is in sleep mode, which leads to an overall improvement in the energy saving of the wireless sensor node while satisfying a specific level of distortion in the estimation problem.

\section{CONCLUSION}

In this paper, we developed a framework for achieving the minimum expected transmit power that satisfies a specific level of distortion in the estimation problem. We obtained the optimal estimator, and derived the optimal transmit power policy by using dynamic programming. The results suggested that one can use a simple threshold event-triggering mechanism [12] for transmission power control with small performance degradation. 


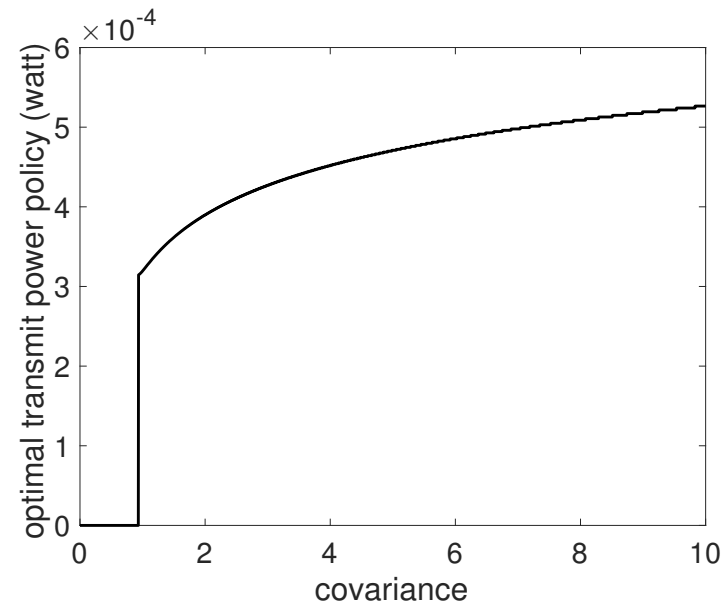

Fig. 2. Near-optimal stationary transmit power policy.

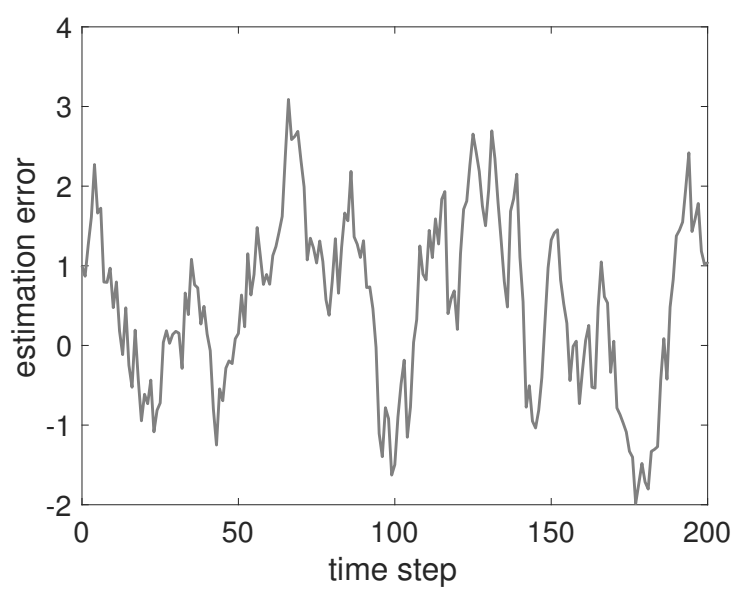

Fig. 3. Trajectory of the estimation error.

\section{ACKNOWLDEGMENT}

This work has been carried out with the support of the Technische Universität München - Institute for Advanced Study, funded by the German Excellence Initiative and the DFG Priority Program SPP1914 "Cyber-Physical Networking". The research of J. S. Baras was also supported by DARPA through ARO grant W911NF1410384, by ARO grant W911NF-15-1-0646, by NSF grant CNS-1544787 and by a Senior Hans Fisher Fellowship from IAS-TUM.

\section{REFERENCES}

[1] I. F. Akyildiz, W. Su, Y. Sankarasubramaniam, and E. Cayirci, "Wireless sensor networks: a survey," Computer networks, vol. 38, no. 4, pp. 393-422, 2002.

[2] N. A. Pantazis and D. D. Vergados, "A survey on power control issues in wireless sensor networks," IEEE Communications Surveys \& Tutorials, vol. 9, no. 4, pp. 86-107, 2007.

[3] B. Sinopoli, L. Schenato, M. Franceschetti, K. Poolla, M. I. Jordan, and S. S. Sastry, "Kalman filtering with intermittent observations," IEEE Trans. on Automatic Control, vol. 49, no. 9, pp. 1453-1464, 2004.

[4] A. S. Leong, S. Dey, G. N. Nair, and P. Sharma, "Power allocation for outage minimization in state estimation over fading channels," IEEE Trans. on Signal Processing, vol. 59, no. 7, pp. 3382-3397, 2011.

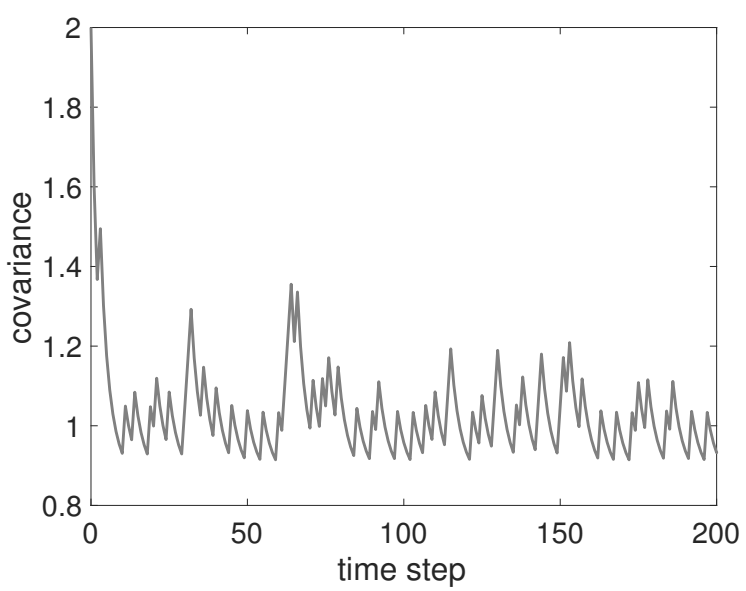

Fig. 4. Trajectory of the estimation error covariance.

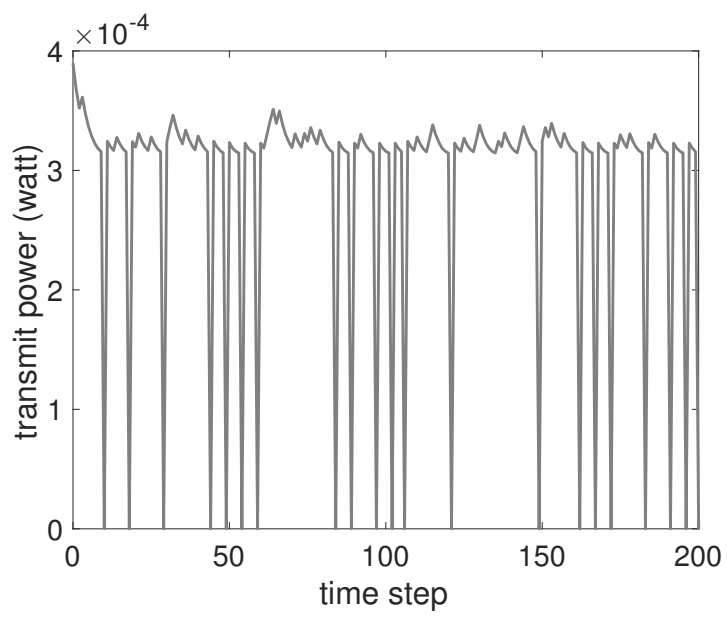

Fig. 5. Trajectory of the transmit power.

[5] J. Wu, Y. Li, D. E. Quevedo, V. Lau, and L. Shi, "Data-driven power control for state estimation: a bayesian inference approach," Automatica, vol. 54, pp. 332-339, 2015.

[6] X. Ren, J. Wu, K. H. Johansson, G. Shi, and L. Shi, "Infinite horizon optimal transmission power control for remote state estimation over fading channels," IEEE Trans. on Automatic Control, vol. 63, no. 1, pp. 85-100, 2018.

[7] Y. Li, F. Zhang, D. E. Quevedo, V. Lau, S. Dey, and L. Shi, "Power control of an energy harvesting sensor for remote state estimation," IEEE Trans. on Automatic Control, vol. 62, no. 1, pp. 277-290, 2017.

[8] M. Nourian, A. S. Leong, and S. Dey, "Optimal energy allocation for kalman filtering over packet dropping links with imperfect acknowledgments and energy harvesting constraints," IEEE Trans. on Automatic Control, vol. 59, no. 8, pp. 2128-2143, 2014.

[9] I. F. Akyildiz and M. C. Vuran, Wireless sensor networks, vol. 4. John Wiley \& Sons, 2010.

[10] K. J. Åström, Introduction to stochastic control theory. Dover Publications, 2006.

[11] D. P. Bertsekas, Dynamic programming and optimal control, vol. 2. Athena Scientific, 1995.

[12] T. Soleymani, S. Hirche, and J. S. Baras, "Optimal stationary selftriggered sampling for estimation," in Proc. of the IEEE Conf. on Decision and Control (CDC), pp. 3084-3089, 2016. 